\title{
Numerical Simulation of the Aerodynamic Influence of Aircrafts During Aerial Refueling with Engine Jet
}

\author{
Yi $\mathrm{Li}^{1}$ (1) $\cdot$ Yang Zhang ${ }^{2}$. Junqiang $\mathrm{Bai}^{1}$
}

Received: 28 December 2018 / Revised: 20 August 2019 / Accepted: 21 August 2019 / Published online: 9 September 2019 (c) The Author(s) 2019

\begin{abstract}
Aerial refueling technology has been widely applied in various fields and it is one of the hotspots but difficulties for the aeronautical technologies. DLR-F6 WBNP model is used as a tanker and a fighter model is used as a receiver. The flow field of Probe-Drogue refueling and Flying Boom refueling is numerically simulated using the Reynolds-averaged Navier-Stokes equations, and the effects of the jet flow and the aerodynamic characteristics of the receiver are taken into consideration. The results indicate that the effect of downwash of the tanker reduces the lift coefficient and decreases the pitching moment coefficient of the receiver. The jet flow of tanker increases the dynamic pressure while decreases the local angle of attack, which increases the pressure difference between the upper and lower surfaces of receiver. Compared with the results without jet, the jet flow can increase the lift and the drag of the receiver and reduces the pitching moment, and even cause the change of rolling moment direction. Therefore, engine jet is an important factor when simulating aerial refueling.
\end{abstract}

Keywords Aerial refueling $\cdot$ Probe-Drogue refueling $\cdot$ Flying Boom refueling $\cdot$ Engine jet $\cdot$ Tanker

\section{Introduction}

Aerial refueling technology plays an important role in the modern warfare, disaster relief and maritime search and rescue. It increases the range and payload of the aircraft and extends the battery life as well as improves the capability of long-range combat or large-scale search and rescue [19].

There are two main methods for aerial refueling: probedrogue aerial refueling and flying boom aerial refueling [15]. For probe-drogue aerial refueling, refueling pod and refueling platform are installed in the fuselage or under the wing. When refueling, the receiving probe located on the nose of the receiver connects with the drogue located at the end of

Yi Li

npuliyi@mail.nwpu.edu.cn

Yang Zhang

youngz@xjtu.edu.cn

Junqiang Bai

junqiang@nwpu.edu.cn

1 School of Aeronautics, Northwestern Polytechnical University, Xi' an 710072, Shaanxi, People's Republic of China

2 State Key Laboratory for Strength and Vibration of Mechanical Structures, Xi'an Jiaotong University, Xi' an 710049, Shaanxi, People's Republic of China the hose which is released by refueling pod or refueling platform. For flying boom aerial refueling, the telescopic boom located at the rear of the tanker is extended to connect with the oil slot located at the top of the receiver.

Because the flight test of aerial refueling is dangerous and the wind tunnel test is expensive, computational fluid dynamics (CFD) has become an important method to simulate aerial refueling in recent years. In 1975, Jewell and Stapleford [10] and Rossow et al. [18] proposed a mathematical model to describe the effect of wake. Modeling and analysis of the effect of a tanker's wake on a receiver aircraft were pioneered by Bloy et al. [1-5], and the results obtained were in good agreement with the wind tunnel experimental data. Venkataramanan and Dogan [7] developed the Vortex Effect Modeling Technique (VEMT) to simulate the flow field of aerial refueling. Jackson et al. [9] used vortex lattice method and CFD method to simulate the flow field of a Learjet flying behind a $\mathrm{KC}-135 \mathrm{R}$ during aerial refueling. The downwash angle predicted by CFD method is closer to the flight test result than the vortex lattice analysis. Euler method was applied to the prediction of the downwash flow field behind a KC-135 tanker investigated by Jurkovich [11]. Euler solutions are sufficient for capturing the tanker flow field for aerial refueling needs. The aerodynamic characteristics of the flow field of an embarked buddy-refueling aircraft 
were analyzed by Yue et al. [21] using Reynolds-Averaged Navier-Stokes (RANS) method, and the basic characteristics of jet blast were derived through the simulation. But the results only included tanker flying solo. Dai et al. [6] present a bow wave effect model and analyses the bow wave effect during probe-drogue aerial refueling. Lofthouse and Nathan [14] used RANS method to generate a dataset of aerodynamic coefficient for a B-52 in aerial refueling formation with a KC-135 tanker. Results showed that, apart from a decrease in lift, there is no significant effect on the forces and moments of a B-52 flying solo and one in aerial refueling formation. But the engine effects of the tanker were not considered. Euler, RANS and Delayed Detached-Eddy Simulation (DDES) methods were used to simulate the flowfield of aerial refueling formation [12]. Jurkovich and Hummer [12] provided a guidance for usage of CFD for obtaining the necessary information while minimizing computational requirements. Although many researchers investigate the aerodynamic interactions between the tanker and receiver, few articles in which engine jet is considered are available.

In the present paper, the CFD method based on NavierStokes equations is used to simulate the aerial refueling, and the aerodynamic interference between tanker and receiver in the different refueling methods is discussed. Meanwhile, through analyzing the effect of engine jet on the aerodynamic characteristics of receiver in detail, we can deem that jet in the air refueling process is an unneglectable factor.

\section{Numerical Method, Geometric Model and Grid}

\subsection{Numerical Method}

The governing equations are the three-dimensional unsteady compressible Reynolds-averaged Navier-Stokes equations. It is expressed as follows (Eq. 1):

$$
\frac{\partial}{\partial t} \iiint_{\Omega} \mathbf{Q} \mathrm{d} V+\iint_{\partial \Omega} F(\mathbf{Q}) \cdot \mathbf{n} \mathrm{d} S=\iint_{\partial \Omega} G(\mathbf{Q}) \cdot \mathbf{n} \mathrm{d} S,
$$

where $\Omega$ is the control volume, $\partial \Omega$ is the boundary of the control volume, $\mathbf{n}$ is the outer normal unit vector of the control surface. $\mathbf{Q}$ is the conserved quantity, $F(\mathbf{Q})$ is the inviscid flux, and $G(\mathbf{Q})$ is the viscosity flux.

The time-dependent conservation law form of the equations is solved using a semi-discrete finite-volume approach with upwind-biasing of the convective and pressure terms and central differencing of the shear stress and heat transfer terms. Roe's Flux Difference Splitting (FDS) scheme [17] has been selected for this study, combined with van Leer's Monotone Upstream-Centered Schemes for Conser-

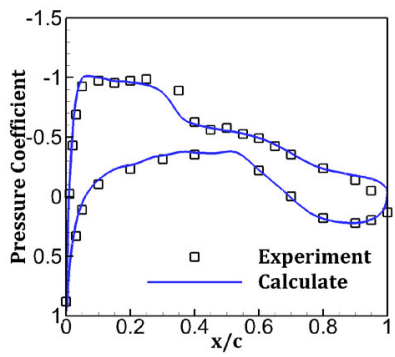

(a) $y / b=0.150$

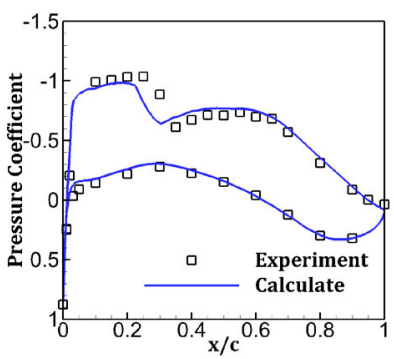

(c) $\mathrm{y} / \mathrm{b}=0.411$

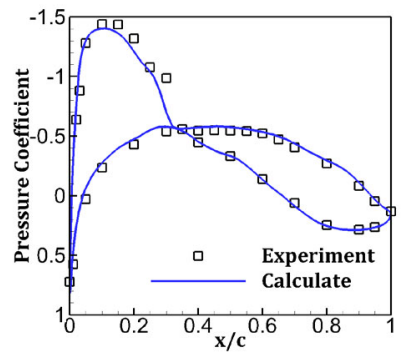

(b) $\mathrm{y} / \mathrm{b}=0.239$

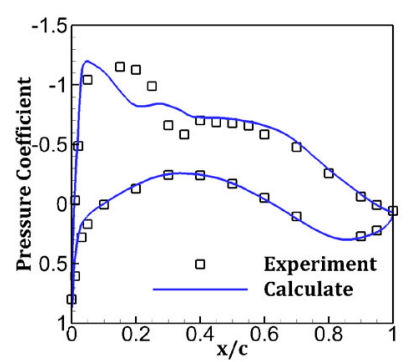

(d) $\mathrm{y} / \mathrm{b}=0.638$
Fig. 1 Pressure coefficient distribution at various spanwise locations for DLR-F6 WBNP

vation Laws (MUSCL) [20] reconstruction at the interface. Besides, the turbulence model used in this study is Menter's $k-\omega$ SST model.

\subsection{Verification of the Flow Solver}

We choose the DLR-F6 wing-body-nacelle-pylon (WBNP) configuration to evaluate the solver and verify its proper implementation. DLR-F6 WBNP is one of the subject geometries in the second AIAA Computational Fluid Dynamics Drag Prediction Workshop [13]. The Mach number of free flow is 0.75 and the Reynolds number based on the mean aerodynamic chord is equal to three million. The lift coefficient is 0.5 . Figure 1 shows the pressure coefficient distribution at various spanwise locations on the wing, where $b$ is the half-span of DLR-F6. In general, the numerical simulation results approximately correspond with the experimental results. This means the flow solver can be used for this study.

\subsection{Geometric Model and Grid}

For the simulation of aerial refueling, the scaled DLR-F6 WBNP configuration is treated as a tanker which can reflect the main components of the tanker. And a fighter model is used as the receiver aircraft. The geometrical parameters of the tanker and receiver are shown in the following table (Table 1). $C_{\text {ref }}$ is the reference chord, $L_{\text {ref }}$ is the reference span and $S_{\text {ref }}$ is the reference area. If the nose of receiver is at original point, the moment reference location is at (16.814, $0,0.35) \mathrm{m}$. 
Table 1 Geometrical parameters for the tanker and receiver

\begin{tabular}{lllr}
\hline & $C_{\text {ref }}(\mathrm{m})$ & $L_{\text {ref }}(\mathrm{m})$ & $S_{\text {ref }}\left(\mathrm{m}^{2}\right)$ \\
\hline Tanker & 4.57 & 42.49 & 151.96 \\
Receiver & 5.37 & 18.14 & 67.71 \\
\hline
\end{tabular}

Table 2 Position of the nose of the aircraft in different aerial refueling methods

\begin{tabular}{lll}
\hline & Receiver $(\mathrm{m})$ & Tanker $(\mathrm{m})$ \\
\hline APD & $(41.02,0.60,-4.95)$ & \\
PPD & $(29.89,13.60,-4.28)$ & $(-12.49,0.00,0.63)$ \\
FB & $(26.84,0.00,-11.44)$ & \\
\hline
\end{tabular}

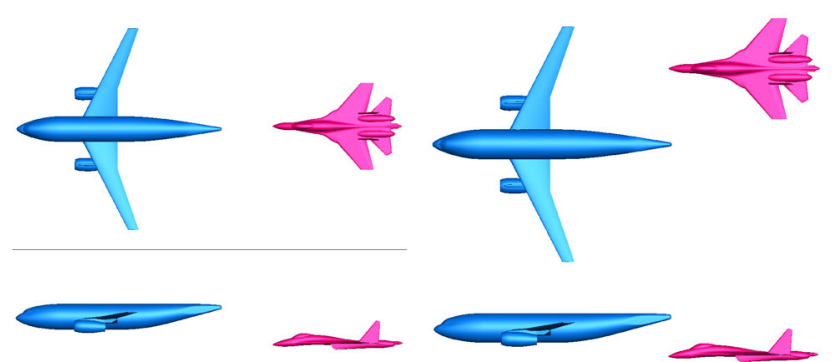

(a) APD

(b) PPD

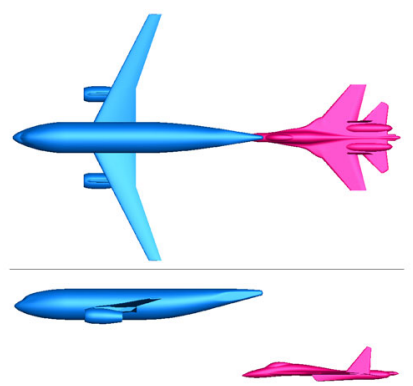

(c) FB

Fig. 2 Position of the tanker (blue) and receiver (purple) during aerial refueling

To take the aerodynamic interference of different aerial refueling position into account, three methods of aerial refueling are simulated. Moreover, as the focus of attention is the aircraft aerodynamic interference and the effect of engine jet, the hose, boom, drogue and other refueling components can be omitted to simplify the calculation. Three aerial refueling methods are afterbody-probe-and-drogue (APD), pod-probeand-drogue (PPD) and Flying boom (FB). Table 2 provides the position of the nose of the tanker/receiver in above three methods.

Figure 2 illustrates the position of the tanker and receiver during aerial refueling, which includes top view and side view of aircrafts. The tanker is blue and the receiver is purple.
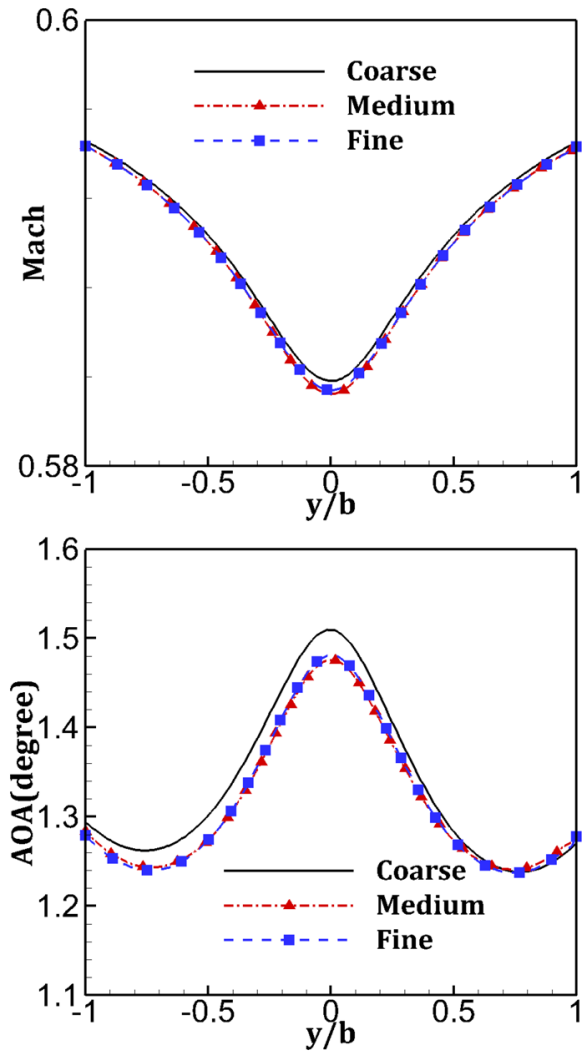

Fig. 3 Distribution of the velocity and the local angle of attack before receiver

For probe and drogue refueling, because the long refueling hose flutters backwards, the horizontal distance between two aircrafts is far and the vertical distance is close. For flying boom refueling, the length of boom is much shorter than the hose, so the distance between two aircraft is closer, but the vertical distance increases.

Computational grids were made using several $\mathrm{O}$ grid topologies. We evaluated the effect of grid refinement. Three grids with different grid level were used in this case: coarse grid (50 million grid points), medium grid (70 million grid points) and fine grid (90 million grid points). Flow field of flying boom aerial refueling with engine jet is simulated with different grids.

A straight line is extract, which is two meters in front of and two meters above the receiver's nose. The distribution of the velocity and the local angle of attack on the line are shown in Fig. 3. The distribution of the velocity of three grids is very close. For local angle of attack, the results calculated by coarse grid are larger than those of other grids. Table 3 shows the results of the receivers for the different grids. Aerodynamic forces and moment obtained by the three grids are very close.

Finally, the medium grid (70 million grid points) is chosen to simulate the flow field of different aerial refueling meth- 
Table 3 Results of the receiver in FB with engine jet for different grids

\begin{tabular}{llll}
\hline Grid & CL & CD & Cm \\
\hline Fine & 0.6493 & 0.09516 & -0.2940 \\
Medium & 0.6480 & 0.09507 & -0.2948 \\
Coarse & 0.6513 & 0.09547 & -0.2901 \\
\hline
\end{tabular}

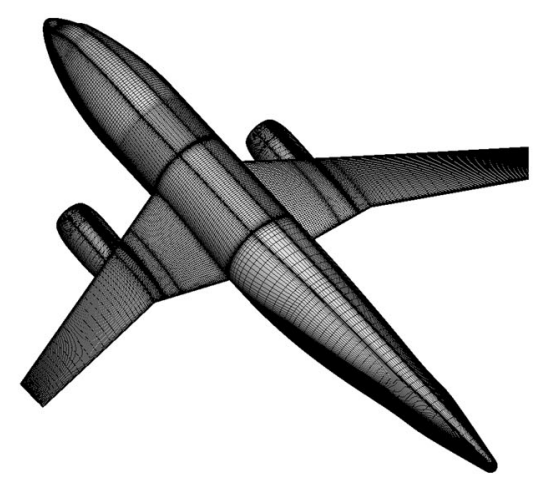

(a) Surface gird of tanker

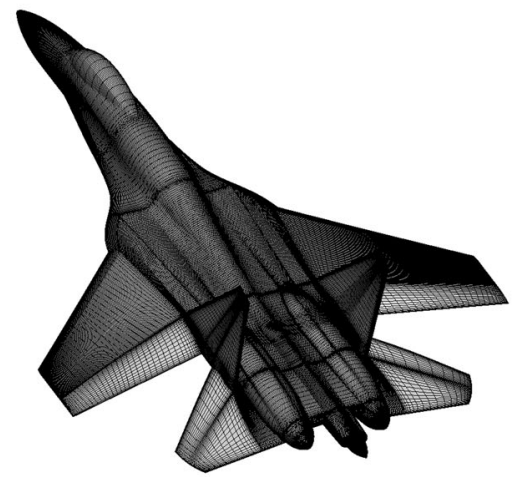

(b) Surface gird of receiver

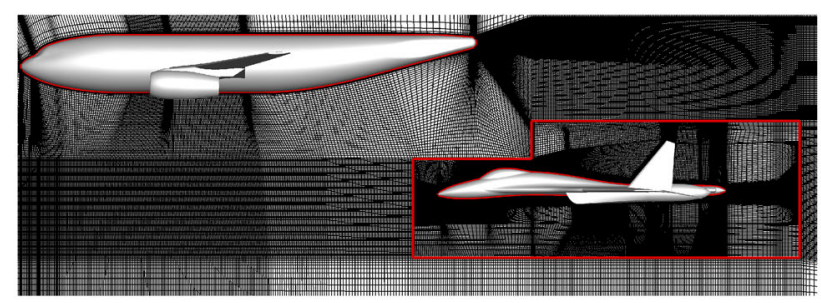

(c) Volume gird for FB

Fig. 4 Surface grid and volume grid for FB

ods, which is the compromise between simulation accuracy and computational cost.

The surface grids of the tanker and receiver and the volume grid for FB are shown in Fig. 4. The patched grid is used. The tanker is in one region and the receiver is in another region. The grid topology of APD and PPD is similar to that of FB. To capture the details of the flow field accurately, the grid is refined at the downstream region of the tanker to reduce the dissipation.

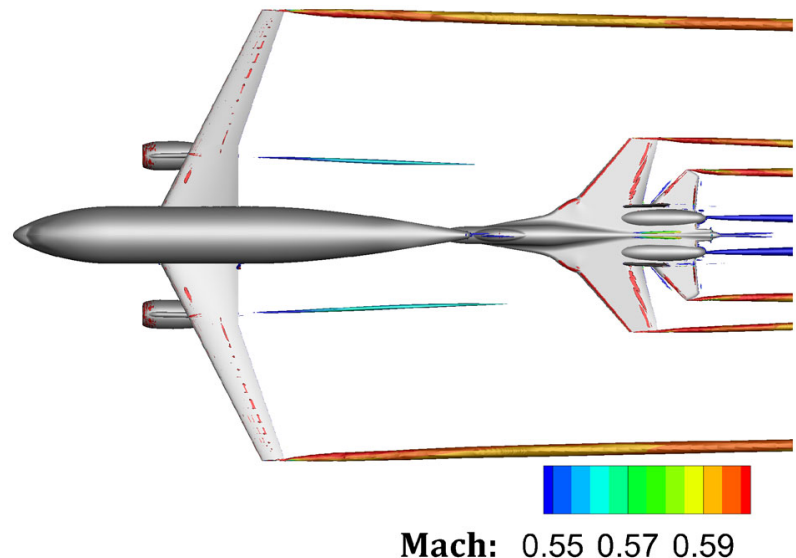

Fig. 5 The contours of $Q$ for FB. $\left(Q=10 s^{-1}\right)$

According to the $\mathrm{Q}$ criterion [8], the contours of $\mathrm{Q}(\mathrm{Q}=$ $10 \mathrm{~s}^{-1}$ ) are shown in Fig. 5. The figure shows that wingtip vortex can be resolved sufficiently beyond the receiver's position. The computational grid is dense enough to well capture the wingtip vortex, wake and jet of tanker.

The internal mechanism of the engine is very complex; however, this paper only take the effect of engine jet into consideration. Therefore, its internal mechanism can be ignored. In this situation, the numerical simulation of the engine power can be replaced by using the inlet and outlet boundary conditions. According to the actual situation, it is subsonic flow at the inlet and outlet boundaries of the engine which can be regarded as the subsonic exit and the subsonic entrance, respectively. According to the characteristic line theory, the engine inlet boundary only needs to be specified a flow parameter, while the engine outlet boundary requires two flow parameters. In the paper, the conditions of the mass flow at the engine inlet and the total temperature and total pressure at the engine outlet are specified. As the mass flow is not the original flow variable, the static pressure at the engine inlet and outlet is adjusted through the iterative process, which will lead the actual mass flow approach to the specified one [16].

\section{Results and Analysis}

\subsection{Simulation Condition}

In this paper, the flight height is $10 \mathrm{~km}$, the freestream pressure is $30,800 \mathrm{~Pa}$, the temperature is $229.733 \mathrm{~K}$, and the flight Mach number is 0.6. The mass flow of the inlet of each engine is $173 \mathrm{~kg} / \mathrm{s}$. The total pressure of the outlet of the engine is $48,200 \mathrm{~Pa}$, and the total temperature is $510 \mathrm{~K}$. The angle of attack of free flow is $2^{\circ}$ during aerial refueling. 
Table 4 Results of the fighter flying solo

\begin{tabular}{llll}
\hline AOA & CL & CD & Cm \\
\hline-1 & 0.449 & 0.06437 & -0.3100 \\
0 & 0.553 & 0.07174 & -0.2876 \\
1 & 0.669 & 0.08273 & -0.2781 \\
2 & 0.778 & 0.09456 & -0.2552 \\
\hline
\end{tabular}

Table 5 Results of the receiver in different aerial refueling methods without jet

\begin{tabular}{lllrrr}
\hline & CL & CD & \multicolumn{1}{l}{ Cl } & \multicolumn{1}{l}{ Cm } & \multicolumn{1}{c}{ Cn } \\
\hline Original & 0.778 & 0.09456 & 0.00000 & -0.2552 & 0.00000 \\
APD & 0.548 & 0.09090 & -0.00089 & -0.3040 & -0.00022 \\
PPD & 0.706 & 0.09580 & -0.00720 & -0.2852 & -0.00281 \\
FB & 0.647 & 0.09491 & 0.00002 & -0.2940 & 0.00002 \\
\hline
\end{tabular}

\subsection{Aerodynamic Performance of the Fighter Flying Alone}

To compare with the aerodynamic performance of receiver during aerial refueling, the situation that the fighter flied alone is simulated firstly. Then, the different aerial refueling methods are simulated and the effect of the engine jet is taken into account. The results of receiver flying solo are shown in Table 4. For the longitudinal static instability of fighter, the pitching moment of receiver increases when the angle of attack increases.

\subsection{Aerodynamic Performance of the Receiver without Engine Jet}

First of all, without engine jet, the aerodynamic interference between the tanker and the receiver is considered. Table 5 shows the aerodynamic results of the receiver flying under different conditions without jet. In Table 5, original represents for the situation that the fighter flies alone. APD, PPD and FB represent for different aerial refueling methods. It is shown that the aerodynamic effect of the tanker on the receiver is different in different aerial refueling methods. Compared with the results of fighter flying alone, the lift coefficient of receiver decreases and the pitching moment increases in aerial refueling. Meanwhile, the drag coefficient decreases in the case of APD, while it increases in the other two refueling methods.

The decrease in pitching moment is due to the downwash effect of the tanker's wake which reduces the local angle of attack at the downstream region of the tanker.

The changes in the aerodynamic characteristics of the receiver in the aerial refueling are shown in Fig. 6. Compared with the results of fighter flying alone, the results of

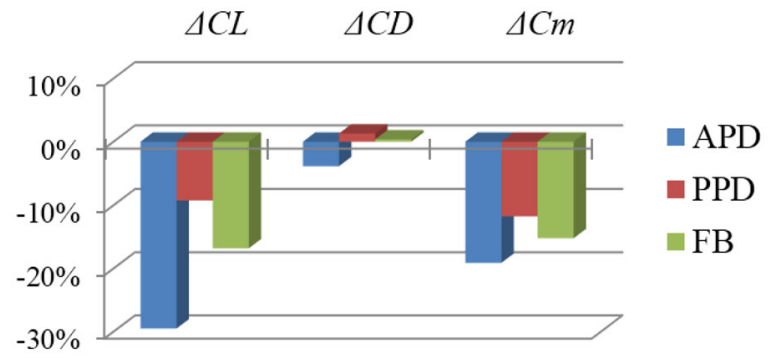

Fig. 6 Changes of the receiver in different aerial refueling methods

aerial refueling without jet show that the change in aerodynamic performance of the receiver in APD is the biggest and the change in PPD is the smallest.

The results shown in Fig. 6 can be summarized as follows:

- In the case of APD, for receiver, the lift coefficient decreases by $29.48 \%$, the drag coefficient decreases by $9.24 \%$ and the pitching moment coefficient decreases by $16.81 \%$. In terms of lift coefficient, the equivalent angle of attack for receiver reduces by $2.06^{\circ}$, which is under the effect of tanker wash. In this method, the wake also changes the rolling and yawing moments, since the receiver is not located directly behind the tanker.

- In the case of PPD, the lift coefficient decreases by $9.24 \%$, the drag coefficient increases by $1.3 \%$, and the pitching moment coefficient decreased by $11.75 \%$. The changes of lift coefficient and pitching moment coefficient of the receiver are the least, and the equivalent angle of attack only reduces by $0.645^{\circ}$.

- In the case of FB, the lift coefficient of the receiver decreases by $16.81 \%$, the drag coefficient increases by $0.36 \%$ and the pitching moment coefficient decreases by $15.21 \%$. The change in drag coefficient is small, and the equivalent angle of attack reduces by $1.17^{\circ}$.

To compare the impact of tanker wake on receiver in three refueling methods, a straight line is extract, which is two meters in front of and two meters above the receiver. Figure 7 shows the distribution of the velocity and the local angle of attack on the line, where $b$ is the half-span of receiver.

As can be seen in Fig. 7, for FB, the distribution of local angle of attack and velocity in the front of receiver is similar to the distribution of the state that receiver flies alone, but there is a decrease in the numerical value. The downwash effect of the wake of the tanker brings a decrease of the local angle of attack and a loss of the flow dynamic pressure. In the case of APD, because the tanker and receiver are closer in horizontal position, there is a great effect of downwash on the receiver. Although the velocity is faster than the distribution of FB on some parts of the line, the local angle of attack is reduced, which leads to a maximum lift loss. In the case of 

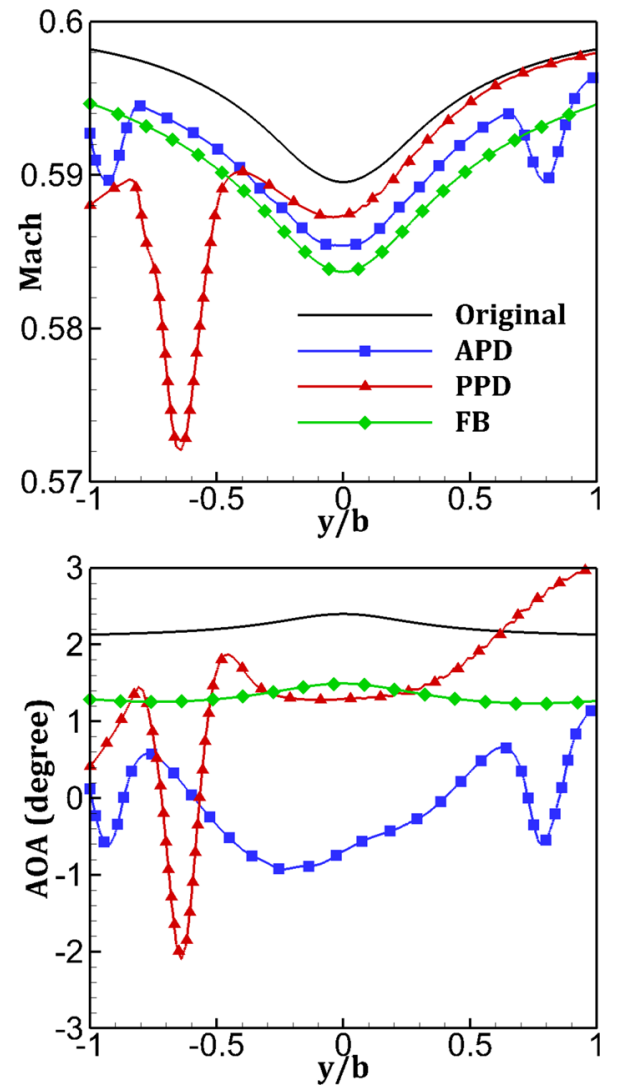

Fig. 7 Distribution of the velocity and the local angle of attack in front of the receiver

PPD, the refueling position is behind the wing of the tanker and the receiver is mainly affected by the wing wake on the one side of the tanker. The distribution of local angle of attack and velocity becomes higher compared with FB and the loss of lift coefficient is the smallest. However, the wing wave of the tanker greatly decreases the rolling moment and yawing moment of the receiver.

Vorticity diagram is extracted in the downstream region of the tanker, and the structure of the wake is shown in Fig. 8. Two pairs of vortex with opposite rotational directions are generated at the tip of tanker wing and downstream region of the nacelle, respectively. In Fig. 8c, the receiver is close to the tanker in the flow direction, but it is fay away from the tanker in the vertical direction during flying boom refueling. Meanwhile, because the receiver is located at the bottom of the trailing vortex of tanker, it is mainly affected by the tanker downwash. While for APD, the distance between two aircrafts is the closest in vertical direction, and the trailing vortex of tanker nacelle sweeps the upper surface of the receiver directly. The indentations on both sides of line for APD in Fig. 7 are caused by the engine trailing vortex. Moreover, for $\mathrm{PPD}$, the receiver is affected by wing tip vortex and engine trailing vortex, which results in large rolling moment and yawing moment.

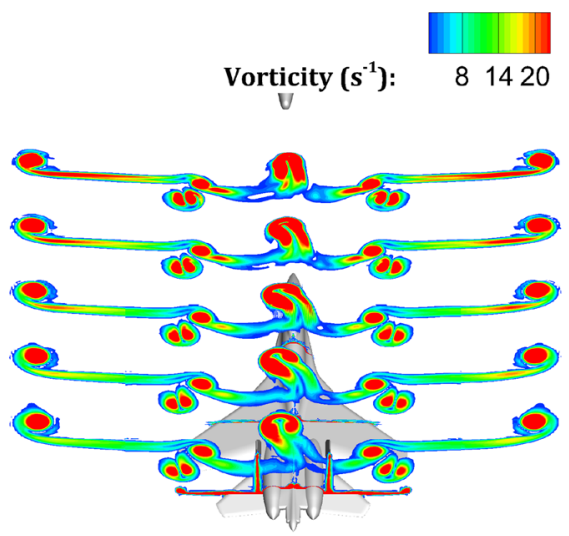

(a) APD

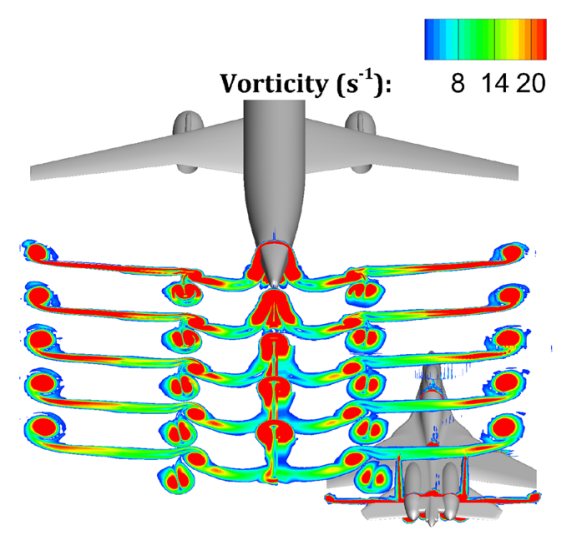

(b) PPD

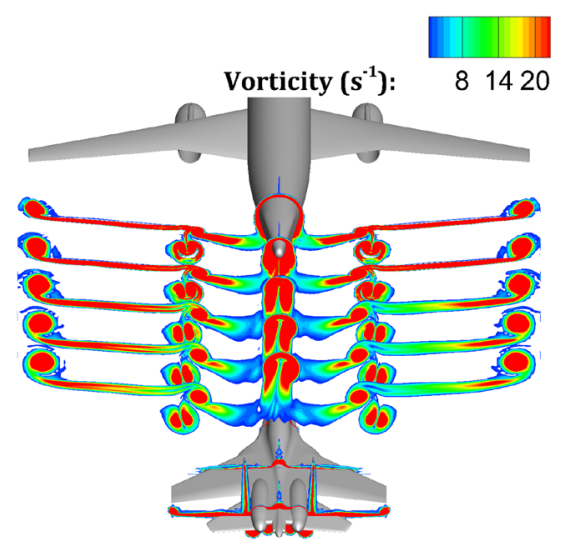

(c) $\mathrm{FB}$

Fig. 8 Vorticity profile during refueling without engine jet

\subsection{The Influence of Engine Jet on the Aerodynamic Performance of the Receiver}

Most of the literatures mainly consider the effect of the wing tip vortex and the wake of the tanker on the receiver, while the influence of engine jet is ignored when the aerial refueling is simulated. In the previous section, the numerical simulation method is used to analyze the aerodynamic interference 


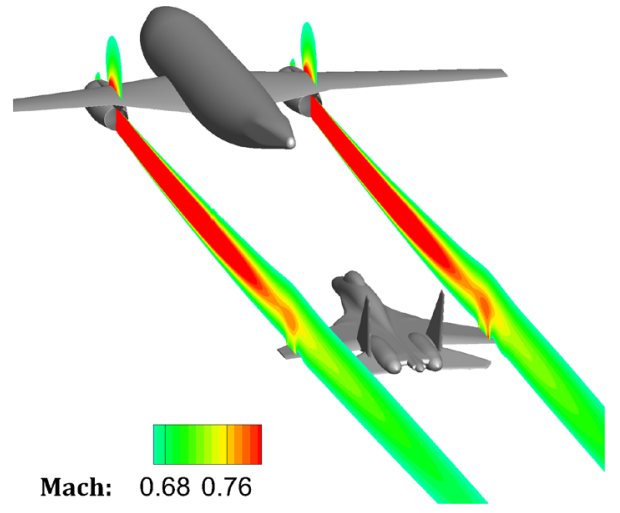

(a) APD

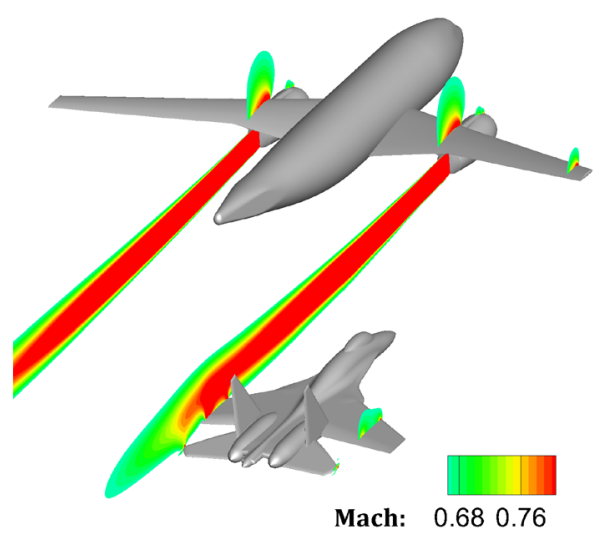

(b) PPD

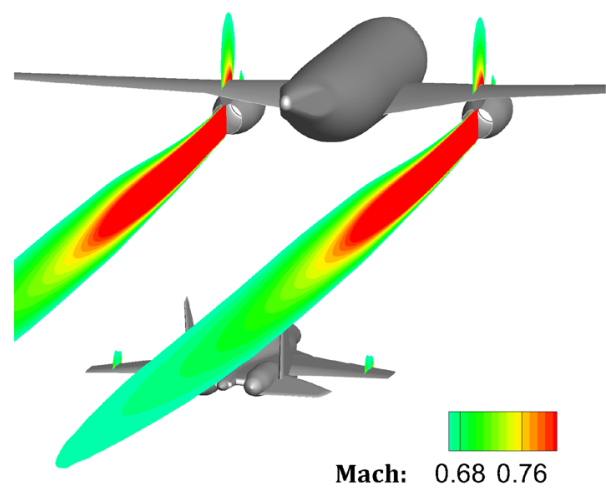

(c) FB

Fig. 9 Velocity profile during refueling with engine jet

between two aircrafts, and it can be seen that the tanker wake does have a great impact on the aerodynamic performance of the receiver. Moreover, through further simulation and comparison, it is found that the impact of tanker engine jet on the aerodynamic performance of the receiver cannot be ignored.

Figure 9 shows the velocity section behind the engine, and only the area where Mach number is greater than 0.66 is shown. The figure also shows the accelerating effect of the engine jet on the flow velocity at downstream region of the engine and near the receiver. It can be seen that the upper
Table 6 Results of the receiver during refueling with engine jet

\begin{tabular}{lllrrr}
\hline & CL & CD & \multicolumn{1}{l}{ Cl } & \multicolumn{1}{l}{ Cm } & \multicolumn{1}{c}{ Cn } \\
\hline Original & 0.778 & 0.09456 & 0.00000 & -0.2552 & 0.00000 \\
APD & 0.576 & 0.09502 & -0.00244 & -0.3193 & -0.00011 \\
PPD & 0.747 & 0.10639 & 0.00068 & -0.3030 & -0.00574 \\
FB & 0.648 & 0.09507 & 0.00000 & -0.2948 & 0.00001 \\
\hline
\end{tabular}

surfaces of the wings on the both sides of the receiver are affected by the accelerating effect of the engine jet in the APD method. In the PPD method, the flow velocity is obviously accelerated on the left wing of the receiver. In the FB method, as the vertical distance between two planes is large and the jet flow passes above the receiver.

Table 6 displays the aerodynamic characteristic data of the receiver with the effect of jet flow. Table 7 shows the comparison between the receiver in the different aerial refueling methods and the fighter flying solo, which contains two cases: with and without jet flow. Overall, compared with the condition without jet, the tanker jet increases the lift coefficient and drag coefficient of receiver, and reduces the pitching moment.

Similarly, intercepting the velocity and the local angle of attack distribution, which is 2 meter ahead of and above the receiver, and the comparison charts are shown in Fig. 10 below. At the rear of the engine of the tanker, the local attack angle is reduced because the jet flow weakens the washing of the tanker. However, the acceleration effect of the jet on the local flow is very obvious, especially in the case of APD and PPD. The strong jet makes the velocity of the intercept position rise greatly, as shown in Fig. 10a.

The angle of attack is positive correlative with the lift coefficient of the aircraft at small angle of attack. From the perspective of the local angle of attack, the decrease in the angle of attack will inevitably lead to the decrease of the lift coefficient of the receiver. However, from the calculation results, the lift coefficient of the receiver with jet is larger than that without jet. This is due to the increase in local dynamic pressure of the jet flow.

From the definition of lift coefficient $C L$ formula (Eq. 2):

$C L=\frac{L}{0.5 \rho_{\infty} V_{\infty}^{2} S_{\mathrm{ref}}}$,

where $\rho_{\infty}$ is the density free flow, $V_{\infty}^{2}$ is the velocity of free flow, and $L$ is the lift of aircraft. The fast-moving jet discharged by engine increases the local dynamic pressure of the fluid in front of the receiver and increases the pressure difference between the upper and lower surfaces of the receiver. Moreover, when the lift coefficient of the receiver is calculated, the dynamic pressure of the free flow is used 
Table 7 Comparison between the receiver in the different aerial refueling methods and the fighter flying solo

\begin{tabular}{|c|c|c|c|c|c|c|}
\hline & \multicolumn{3}{|l|}{ Jet } & \multicolumn{3}{|l|}{ Nojet } \\
\hline & CL (\%) & $\mathrm{CD}(\%)$ & $\mathrm{Cm}(\%)$ & CL (\%) & $\mathrm{CD}(\%)$ & $\mathrm{Cm}(\%)$ \\
\hline APD & -25.95 & 0.48 & -25.10 & -29.48 & -3.87 & -19.10 \\
\hline PPD & -3.96 & 12.51 & -18.72 & -9.24 & 1.30 & -11.75 \\
\hline FB & -16.68 & 0.53 & -15.51 & -16.81 & 0.36 & -15.21 \\
\hline
\end{tabular}

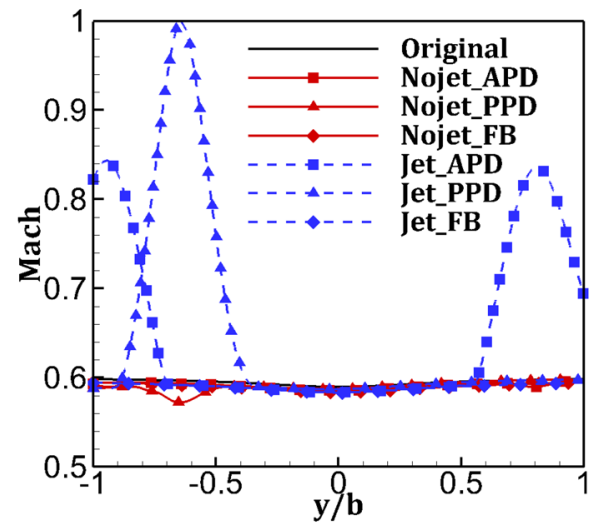

(a) Comparison of the velocity distribution

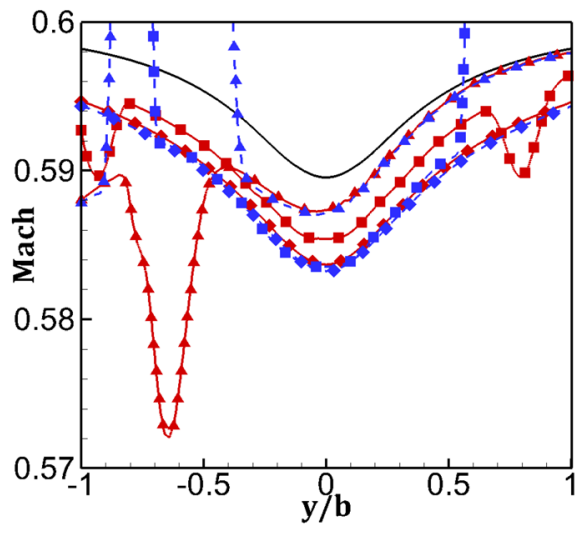

(b) Comparison of velocity distribution

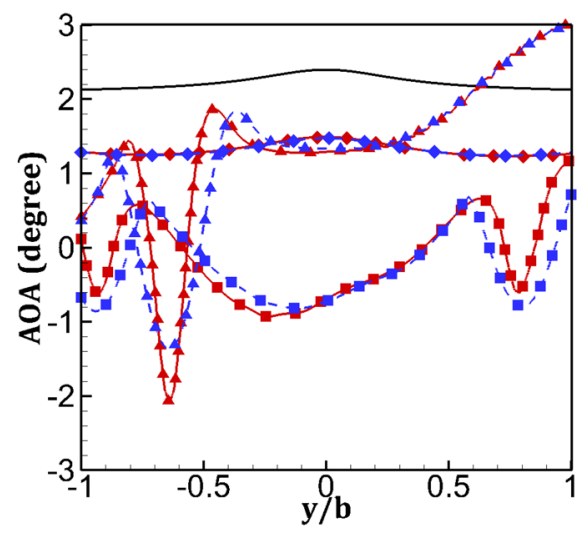

(c) Comparison of the local angle of attack distribution

Fig.10 Distribution of the velocity and the local angle of attack in front of receiver

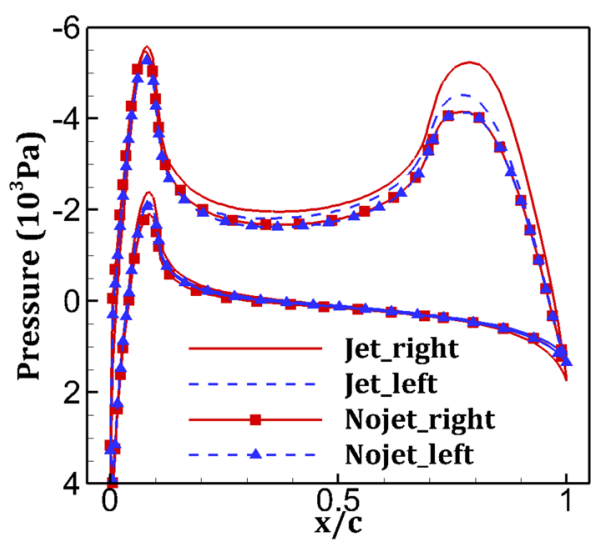

(a) APD

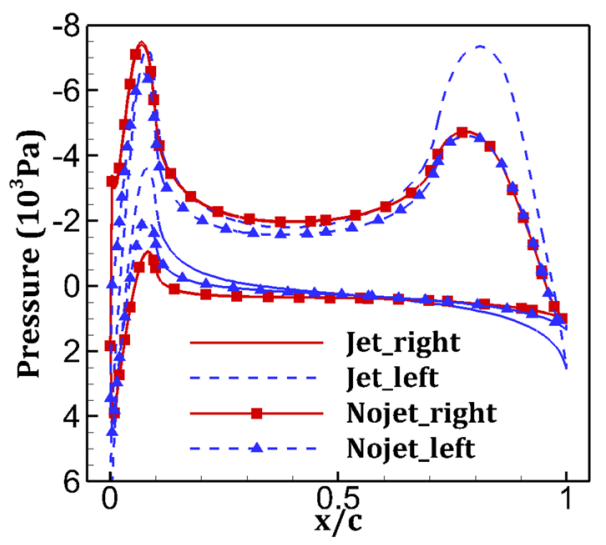

(b) PPD

Fig. 11 Pressure distribution of the section on the receiver's wings

to nondimensionalize the parameter, which naturally leads to the increase of lift coefficient.

The section is extracted on the wing of the receiver to obtain the pressure coefficient distribution. The section is parallel to the symmetry plane of the receiver. For APD, the section is $7 \mathrm{~m}$ away from the plane of symmetry, while it is $5 \mathrm{~m}$ for PPD. The following figure is obtained. In Fig. 11, compared with no-jet condition, the pressure difference between the upper and lower surfaces of the receiver is increased for jet condition. Then the lift increases. The pressure on the upper side of the left wing decreases more for PPD, because the tanker jet flows through the left wing only.

Table 8 shows the rolling/yawing moment coefficient of the receiver. Figure 12 shows the sections of vorticity of the 
Table 8 Results of the receiver in different aerial refueling methods

\begin{tabular}{lrrrrr}
\hline & \multicolumn{1}{c}{ Jet } & & \multicolumn{1}{l}{ Nojet } \\
\cline { 2 - 3 } \cline { 5 - 6 } & \multicolumn{1}{c}{$C n$} & & \multicolumn{1}{c}{$C n$} & \multicolumn{1}{c}{$C n$} \\
\hline APD & -0.00244 & -0.00011 & & -0.00089 & -0.00022 \\
PPD & 0.00068 & -0.00574 & & -0.00720 & -0.00281 \\
FB & 0.00000 & 0.00001 & & 0.00002 & 0.00002 \\
\hline
\end{tabular}

tanker wake with jet flow. It clearly shows the process that the wing vortex and the trailing vortex of the pod in the wakes of the tanker pass through the receiver.

In the case of APD, the tanker's wake flows through the outer side of the vertical tail of the receiver and interferes with the wing and tail. Meanwhile, because the receiver is located in the right rear of the tanker, the right wing and tail are affected by the jet more strongly. In Fig. 7a, the pressure difference between the upper and lower surfaces of the wing on the right side of the receiver is greater and the lift is higher, so that the rolling moment of the receiver is reduced.

In the case of PPD, the receiver is affected by the tail vortex of the tanker and wing tip vortex. The rolling moment of the receiver is a negative value without jet and it becomes positive when the jet flow of the tanker is considered. In comparison of Figs. 8b, 9b and 12b, it can be found that the decay flow velocity which results from the nacelle wake decreases the negative pressure peak at the leading edge of the receiver's left wing, and the pressure difference between upper and lower surfaces is decreased. The lift provided by right wing is greater than that of left wing, and the rolling moment of the receiver is negative. The acceleration effect of the jet changes this situation when the tanker jet is considered. It reduces the pressure near the trailing edge of the upper surface of the receiver's left wing and the lift provided by left wing is more than that of right wing. Therefore, the rolling moment of the receiver becomes positive.

In the case of FB, the same effect of jet flow on both sides of the receiver is observed because the receiver is located just behind the tanker. Moreover, the jet flow has little effect on the rolling/yawing moment coefficient of the receiver.

Above all, the jet flow of tanker has a significant impact on the aerodynamic characteristics of the receiver. Although the aerodynamic characteristics of receiver are mainly affected by the wave of tanker, the jet flow is still a factor that cannot be ignored in the design of aerial refueling system.

\section{Conclusion}

Based on the numerical simulation, the aerodynamic characteristics of receiver in the different aerial refueling methods

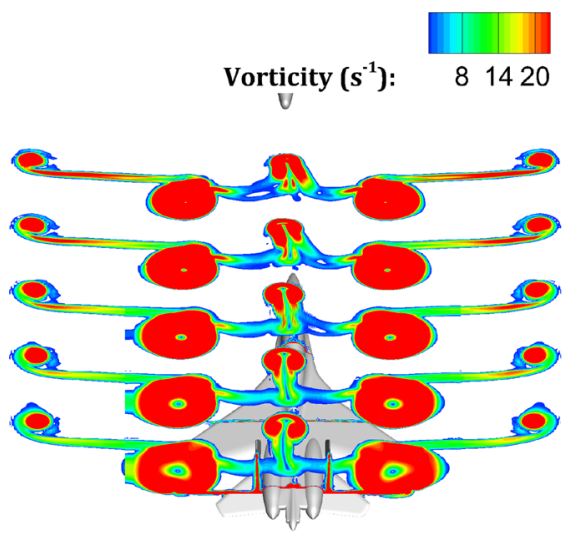

(a) APD

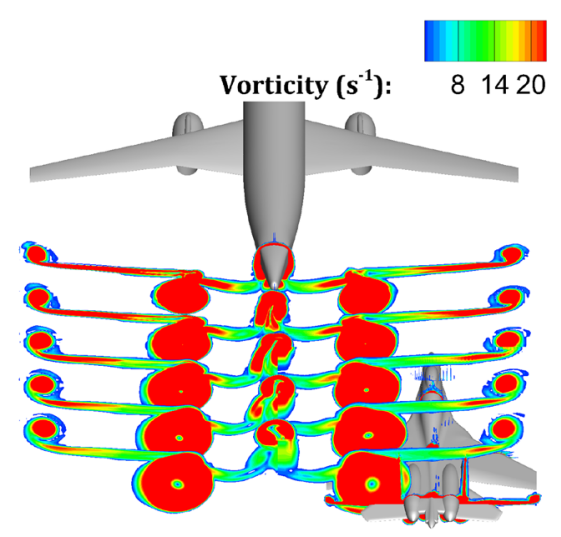

(b) PPD

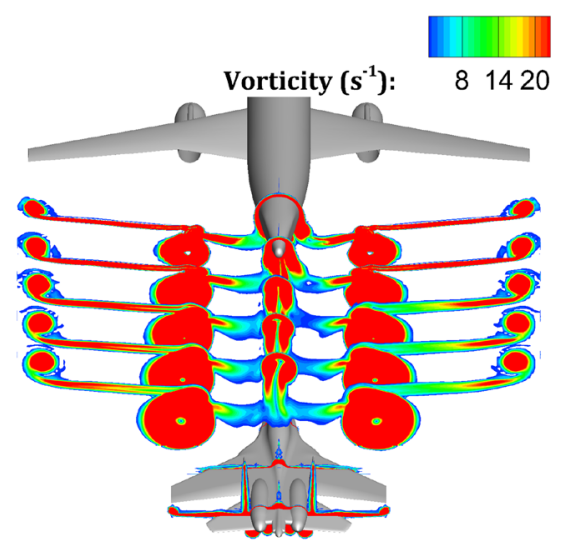

(c) FB

Fig. 12 Vorticity profile during refueling with engine jet

are studied and the effect of jet flow is considered. The following conclusions are obtained:

During aerial refueling, the lift coefficient of receiver decreases for the washing of tanker and the pitching moment coefficient decreases. The drag coefficient varies with different aerial refueling methods.

Compared with the results without jet, the lift coefficient and drag coefficient of the receiver increase and the pitching 
moment of the receiver decreases with jet flow. The main effect of the tanker jet accelerated the flow in front of the receiver and increased the local dynamic pressure.

The jet flow changes the magnitude of the rolling and yawing moment. And the changes of moments affect the flight attitude of receiver. Compared with the results without jet, the deflected direction of the receiver's rudder is changed when the jet of tanker is considered.

Overall, the impact of the jet flow on the aerodynamic characteristics of the receiver cannot be ignored. In the design of aerial refueling system, the jet flow should be taken into consideration.

Open Access This article is distributed under the terms of the Creative Commons Attribution 4.0 International License (http://creativecomm ons.org/licenses/by/4.0/), which permits unrestricted use, distribution, and reproduction in any medium, provided you give appropriate credit to the original author(s) and the source, provide a link to the Creative Commons license, and indicate if changes were made.

\section{References}

1. Bloy A, Lamont P, Abu-Assaf H, Ali K (1986) The lateral dynamic stability and control of a large receiver aircraft during air-to-air refuelling. Aeronaut J 90(896):237-243

2. Bloy A, Trochalidis V (1990) The aerodynamic interference between tanker and receiver aircraft during air-to-air refuelling. Aeronaut J 94(935):165-171

3. Bloy A, Trochalidis V, West M (1991) The aerodynamic interference between a flapped tanker aircraft and a receiver aircraft during air-to-air refuelling. Aeronaut J 95(948):274-282

4. Bloy A, West M (1994) Interference between tanker wing wake with roll-up and receiver aircraft. J Aircr 31(5):1214-1216

5. Bloy A, West M, Lea K, Jouma'a M (1993) Lateral aerodynamic interference between tanker and receiver in air-to-air refueling. $\mathrm{J}$ Aircr 30(5):705-710

6. Dai X, Wei Z, Quan Q (2016) Modeling and simulation of bow wave effect in probe and drogue aerial refueling. Chin $\mathrm{J}$ Aeronaut 29(2):448-461

7. Dogan A, Venkataramanan S, Blake W (2005) Modeling of aerodynamic coupling between aircraft in close proximity. J Aircr 42(4):941-955
8. Hunt JC, Wray AA, Moin P (1988) Eddies, streams, and convergence zones in turbulent flows. Technical Report CTR-S88, Center for Turbulence Research

9. Jackson D, Tyler C, Blake W (2007) Computational analysis of airto-air refueling. In: 25th AIAA applied aerodynamics conference, p 4289

10. Jewell W, Stapleford R (1975) Mathematical models used to simulate aircraft encounters with wake vortices. Technical Report STI TR-1035-4 (DOT-FA73WA-3276-1), NASA

11. Jurkovich M (2011) Cfd prediction of the flowfield behind the kc135r tanker. In: 29th AIAA applied aerodynamics conference, $\mathrm{p}$ 3510

12. Jurkovich MS, Hummer CJ (2018) Understanding the flowfield behind a tanker aircraft. In: 2018 AIAA aerospace sciences meeting, p 536

13. Laflin KR, Klausmeyer SM, Zickuhr T, Vassberg JC, Wahls RA, Morrison JH, Brodersen OP, Rakowitz ME, Tinoco EN, Godard JL (2005) Data summary from second aiaa computational fluid dynamics drag prediction workshop. J Aircr 42(5):1165-1178

14. Lofthouse A.J, Nathan B (2017) CFD modeling of B-52 and KC135 in air refueling formation. In: 35th AIAA applied aerodynamics conference, $\mathrm{p} 4236$

15. Lu Y, Yang C, Liu Y (2014) A survey of modeling and control technologies for aerial refueling system. ACTA Aeronaut Astronaut Sin 35(9):2375-2389

16. Qiao L, Bai J, Hua J et al (2014) Interference effects of wing mounted high bypass ratio nacellle with engine power. ACTA Aerodyn $\operatorname{Sin} 32(4): 433-438$

17. Roe $P$ (1981) Approximate riemann solvers, parameter vectors, and difference schemes. J Comput Phys 43(2):357-372

18. Rossow VJ, Corsiglia VR, Schwind RG, Frick JK, Lemmer OJ (1975) Velocity and rolling-moment measurements in the wake of a swept-wing model in the 40 by 80 foot wind tunnel. Technical Report TM X-62414, NASA

19. Thomas PR, Bhandari U, Bullock S, Richardson TS, du Bois JL (2014) Advances in air to air refuelling. Prog Aerosp Sci 71:14-35

20. Van Leer B (1979) Towards the ultimate conservative difference scheme. V. A second-order sequel to Godunov's method. J Comput Phys 32(1):101-136

21. Yue K, Cheng L, Zhang T, Ji J, Yu D (2016) Numerical simulation of the aerodynamic influence of an aircraft on the hose-refueling system during aerial refueling operations. Aerosp Sci Technol 49:34-40

Publisher's Note Springer Nature remains neutral with regard to jurisdictional claims in published maps and institutional affiliations. 